\title{
From medical data to simple virtual mock-up of scapulo-humeral joint
}

\author{
H.ATMANI ${ }^{*}$, F.MERIENNE* ${ }^{*}$ D.FOFI ${ }^{*}$ and P.TROUILLOUD ${ }^{+}$ \\ * Insitut image-ENSAM, Le2i, rue thomas Dumorey, Chalon sur saone, France \\ * IUT du Creusot, Le2i, rue de la fonderie, Le Creusot, France \\ +University of Burgundy, Laboratoire d'anatomie, Dijon, France
}

Received $14^{\text {th }}$ May 2008; accepted $8^{\text {th }}$ June 2009

\begin{abstract}
The surgical operations of shoulder joint are guided by various principles: osteosynthesis in the case of fracture, osteotomy in order to correct a deformation or to modify the functioning of the joint, or implementation of articular prosthesis. At the end of the twentieth century, many innovations in the domains of biomechanics and orthopedic surgery have been performed. Nevertheless, theoretical and practical problems may appear during the operation (visual field of surgeon is very limited, quality and shape of the bone is variable depending on the patient). Biomechanical criteria of success are defined for each intervention. For example, the installation with success of prosthetic implant will be estimated according to the degree of mobility of the new articulation, the movements of this articulation being function of the shape of the prosthesis and of its position on its osseous support. It is not always easy to optimize the preparation of the surgical operation for every patient, and a preliminary computer simulation would allow helping the surgeon in its choices and its preparation of the intervention. The techniques of virtual reality allow a high degree of immersion and allow envisaging the development of a navigation device during the operating act.
\end{abstract}

Key Words: Shoulder, 3D modeling, medical imaging, virtual surgery, image processing.

\section{Introduction}

The context of this work is related to the operation of the replacement of shoulder articulation by a prosthesis. The substitution of the articulations of the knee and the hip by prostheses is a procedure which succeeds well. On the other hand, the case of the prosthesis of shoulder is much less obvious. The main cause of failure of the positioning of the prosthesis is related to the anatomy of the shoulder1. The visual field of the surgeon is very limited during the operation because a small incision is carried out on the patient to limit the damage to surrounding tissues. Consequently, only the sleeve of the scapula and the humeral head are exposed. The goal of this work is the development of a virtual reality system in order to simplify the preoperative and peroperative work for surgeons when substituting the shoulder articulation by prosthesis. To reach this goal, different steps have been identified:

- Modelling the bones of human shoulder from available medical images of the patient.

Correspondence to: 〈hakim.atmani@univ-tln.fr>

Recommended for acceptance by <Ralph Seulin> ELCVIA ISSN: 1577-5097

Published by Computer Vision Center / Universitat Autonoma de Barcelona, Barcelona, Spain 
- Simulation of the operation from this model called virtual object (preoperative virtual surgery).

- Designing of a real-time augmented reality system for peroperative work.

The Fig. 1 describes the proposed method to reach the presented goal.

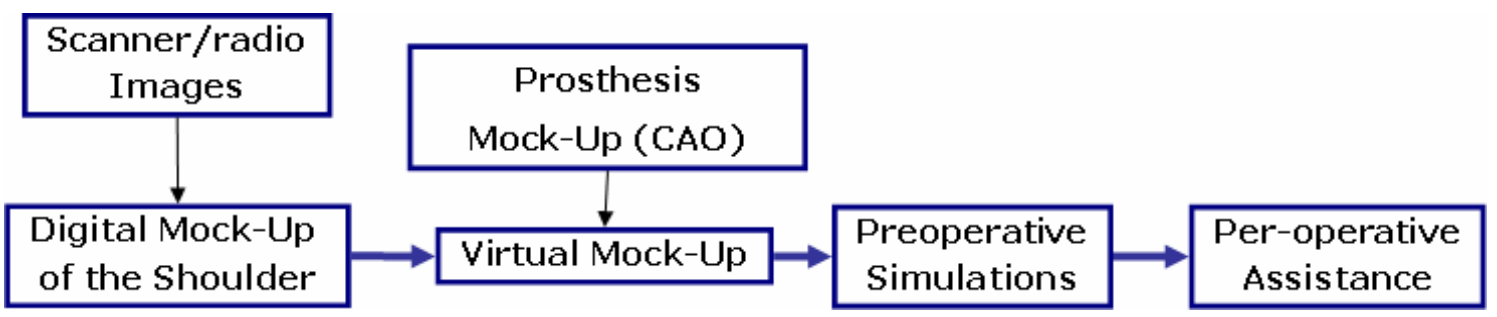

Figure 1. Towards a computer-aided surgery system for the shoulder prosthesis placement.

\section{State of the art}

The purpose of the virtual object in each CAS (computer aided surgery) application is to provide a realistic representation of the bony structures or organs that are involved in the intervention. Acquisition of virtual objects (VOs) may be achieved either preoperatively or intraoperatively. Approximately a decade ago, the first computer assisted orthopaedic surgery (CAOS) systems were introduced based on preoperatively acquired CT scans. The advantage of such a system is that it offers excellent contrast between bone and soft tissue. Moreover, the acquired images are geometrically undistorted and thus no sophisticated calibration is required. These advantages make CT images superior to MRI as preoperative VOs, although the latter method has clear advantages in terms of the patient's radiation exposure. Some efforts have been made to overcome MRI-related difficulties [2,3], but CT remains the method of choice for preoperative imaging for CAOS applications. Another drawback of preoperative VOs led to the introduction of intraoperative imaging modalities. The bony morphology may change between image acquisition and performance of the actual surgical procedure. Consequently, the VO may not necessarily correspond to the therapeutic object, leading to unpredictable inaccuracies during navigation or robotic procedures. This effect can be particularly problematic for traumatology in the presence of unstable fractures. To overcome this difficulty, the use of intraoperative CT scanning has been proposed4, but the infrastructural changes required for implementation of this approach are tremendous, often involving considerable reconstruction of a hospital's facilities5. An alternative approach is the use of established intraoperative imaging modalities. Several research groups have developed navigation systems based on fluoroscopic images [4,5]. The fluoroscope is a well-established device in orthopaedic and trauma treatment, and could therefore be integrated into CAOS systems more easily than intraoperative CT. In contrast to CT, however, images generated with a fluoroscope are usually distorted due to a number of factors. To use fluoroscopic images as VOs therefore requires calibration of the fluoroscope, which involves the attachment of marker grids to the image intensifier and the tracking of its position and orientation with the navigator during image acquisition [6,7]. The resulting real-time visual feedback provided by the navigation system is similar to the use of the fluoroscope in constant mode. This technique is therefore also known as "virtual fluoroscopy" [8]. Although only 2D projections are available and the images usually lack contrast when compared to preoperative CT, the advantages of fluoroscopybased navigation preponderate for a number of clinical applications. Recently, a novel imaging device has been developed [9] that enables the intraoperative generation of 3D fluoroscopic image data. It

consists of a motorized, isocentric C-arm that acquires series of 50-100 2D projections and from them reconstructs $13 \times 13 \times 13 \mathrm{~cm}$ volumetric datasets that are comparable to CT scans. Initially advocated primarily for surgery on the extremities, this "fluoro-CT" has been adopted for use with a navigation system and has already been applied to several anatomic areas (see Clinical fields of application below). One major advantage of the device is that it combines the availability of 3D imaging with intraoperative data acquisition. A final category of navigation systems functions without using any radiological images as VOs. 
Instead, the tracking capabilities of the system are used to acquire a graphic representation of the patient's anatomy by intraoperative digitization. Using any tracked instrument, the spatial location of anatomic landmarks can be recorded. Combining the obtained points into lines and surfaces will generate, step by step, an abstract model of the geometry. As this model is generated by the operator, the procedure is known as "surgeondefined anatomy" (SDA). The technique is particularly useful when soft tissue structures such as ligaments or cartilage boundaries are to be considered that are difficult to identify on CT or fluoroscopic images. Moreover, some locations can be acquired without direct access by a digitizing instrument. For instance, the center of the femoral head, which is an important landmark during total knee replacement, can be reconstructed from recorded passive rotation of the leg around the acetabulum. However, the generated images are often rather abstract and not easy to interpret. Sati et al. [10] suggested underlaying a preoperative X-ray to facilitate orientation, but precise matching of the two image spaces turned out to be difficult. An alternative concept is provided by the so-called bone morphing technique $[11,12]$. This process uses a database of generic 3D statistical computer models of bones and a set of specific points that are acquired with the SDA technique. Analyzing the recorded data enables the system to select the bone model from the data pool that best matches the patient's morphology. A special morphing algorithm then deforms the selected model three-dimensionally until it fits the acquired points as perfectly as possible. As a result, a realistic virtual model of the structure to be operated on can be presented and used as a VO without any conventional image acquisition.

In our work, we started with the idea that $\mathrm{CT}$ scans are not always available, so our shoulder model can be parameterized using X-rays or CT. For the application, it is not necessary to perform accurate modeling of a real shoulder joint: a model based on simple forms is sufficient for measuring the prosthetized shoulder.

\section{Shoulder anatomy}

The shoulder is a set of joints which allow the hand to orient on a wide area of space; this volume is greater than a halfsphere. It is the most mobile joint in the body but this great mobility have a corollary: a big instability that provides most of mechanical pathologies. The shoulder is composed of the humeral head and the articular cavity of the scapula or glenoid, covered by the articular cartilage. The scapula extends to form the bony excrescence: the acromion surrounds the shoulder from backward to forward to form its roof, and the coracoid is forward (Fig. 2).

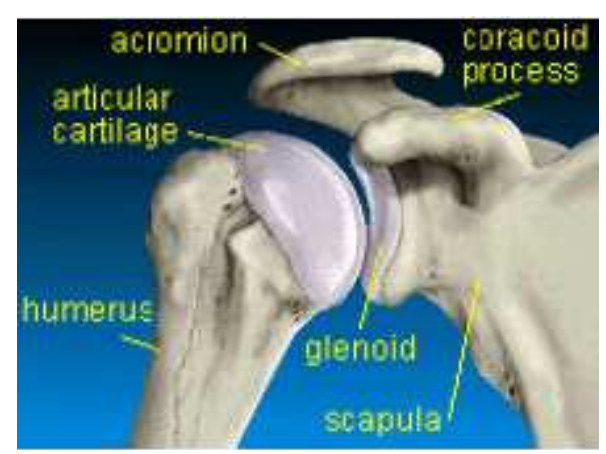

Figure 2. Bony part of shoulder.

\section{Digital mock-up}

\subsection{Comparison of different techniques for shoulder modelling}

With respect to the final application (computer-assisted surgery), different criteria are necessary in order to determine the best possible modelling method:

- Parameterizable surface, which allows us to adapt the surface to the morphological characteristics of the patient, 
- Parameter type, because a surface ruled by physical parameters consents to deform the model morphologically which is not the case with mathematical parameters,

- Logical operators, because the surface has to allow logical operations to ease the subsequent virtual surgery.

Different techniques from the literature have been implemented and evaluated ([13] to [23]). The Table 1 resumes the obtained results according to the mentioned criteria.

\begin{tabular}{ccccc}
\hline $\begin{array}{c}\text { Implemented } \\
\text { methods }\end{array}$ & Surface/Volume? & Parameters & $\begin{array}{c}\text { Kind of } \\
\text { parameters }\end{array}$ & $\begin{array}{c}\text { Operators } \\
\text { (intersection, } \\
\text { substraction, union) }\end{array}$ \\
\hline RBF & Surface & Yes & Mathematical & Possible \\
\hline Kohonen & Surface & Yes & Mathematical & Possible \\
\hline Skeleton & Surface & No & - & Impossible \\
\hline Snakes & Surface & No & - & Impossible \\
\hline Subdivision & Surface & No & - & Possible \\
\hline Marching & Volume or & No & - & Possible \\
\hline Simple-form & Surface & Surface & Yes & Morphological \\
\hline
\end{tabular}

Table 1: Comparison of the methods

RBF, Kohonen maps and simple-form modeling methods are parametric representations. Nevertheless, for RBF and Kohonen maps, the parameters are purely mathematics and do not represent any anatomical information (such as the diameter of the humeral head or the length of the humerus). Moreover, it seems quite difficult to determine anatomical information by combining the mathematical parameters of these representations. All the other methods are nonparametric so that the shape and size of the reconstruction can hardly be adapted to the patient morphology unless a morphing algorithm is applied. The chosen modelling method is the simple-form one. In that model, the bones of the shoulder are represented by simple forms, such as quadrics, planes and so on, that can be handled with a very few parameters. It has been already stated that the data provided by the surgeon are sufficient to parameterize this kind of model. Furthermore, logical operations as subtraction or intersections can be easily computed on quadrics and planes so that the drilling and cutting can be simulated on the model and a virtual operation can be considered.
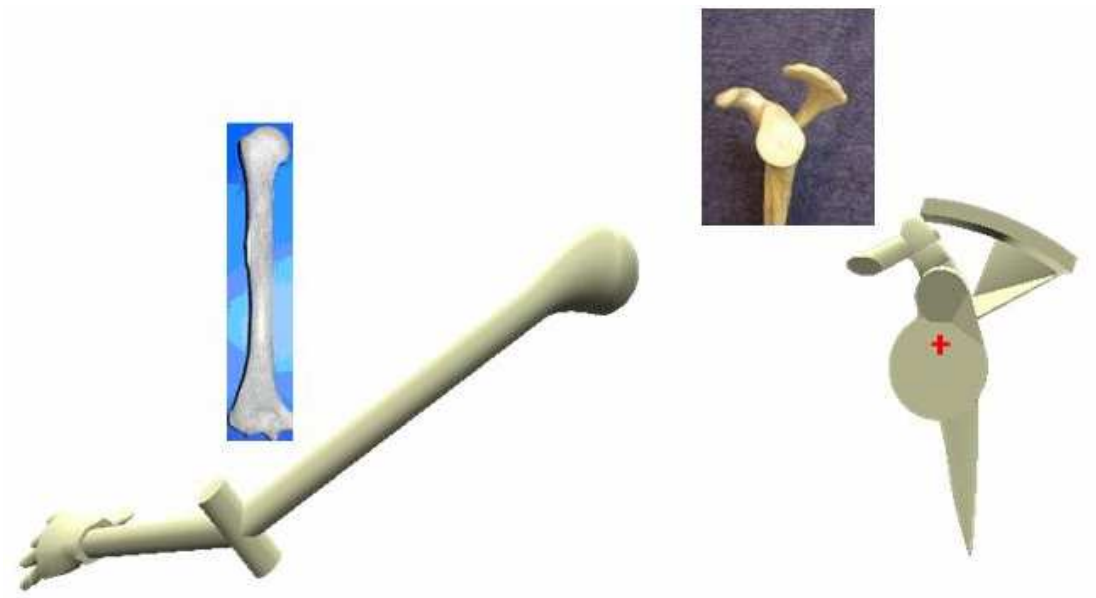

Figure 3. The real shoulder with simple-form model developed. 
It is important to notice that the model is used in the context of the replacement of the bones articulation by a prosthesis articulation. In our case, the inverse prosthesis which is the DUOCENTRIC prosthesis is installed. Then, for the application, it is not necessary to perform a fine model of a real shoulder articulation. The simple-form model is sufficient for the application. The simple-form model proposed is composed of quadrics, boxes and Dupin supercyclids for the links between different forms. For instance, the humerus is mainly made of a sphere and a cylinder [24]. A Dupin supercyclid is added to ensure the continuity and completeness of the model and the bicipital groove is modelled by carving a cylinder into the humeral head. The scapula is composed of planes, ellipsoids, cylinders and so on. The complete model will be detailed and the link between relevant parameters and simple form parameters that legitimates our choice will be stated.

Then, only 14 main parameters are necessary to compute a model adapted to the morphology of the patient. In accordance with orthopaedists [25], the parameters of interest described in the Table 2 have been chosen.

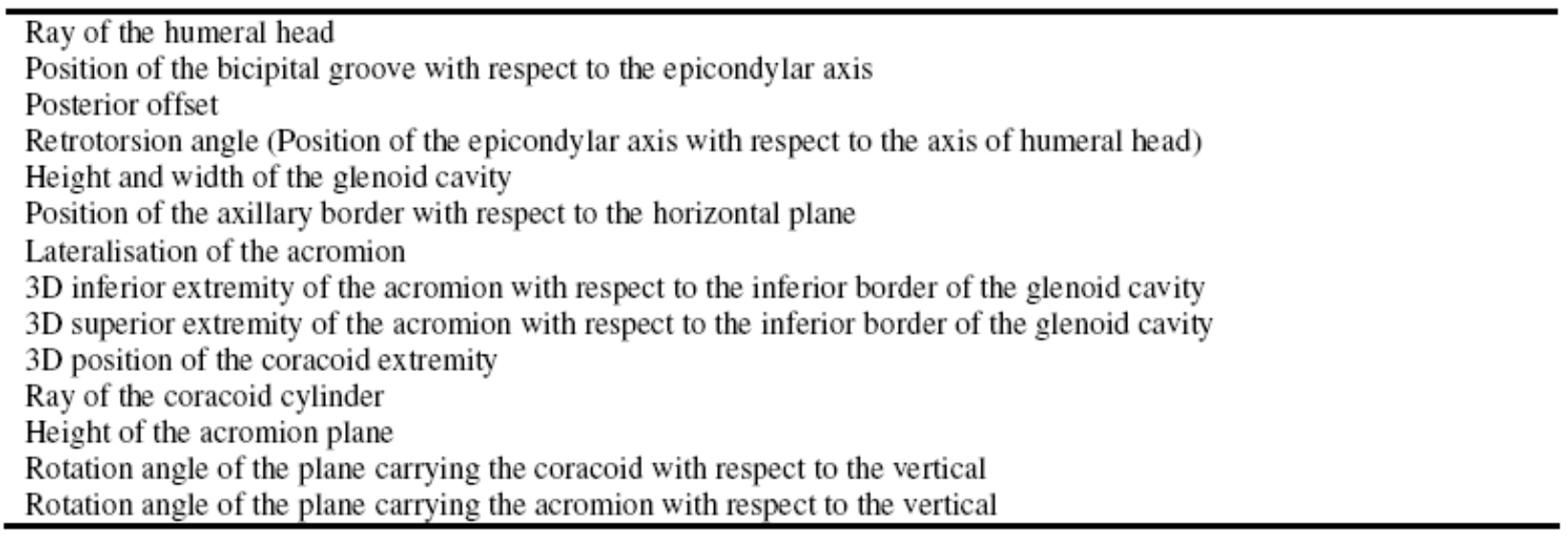

Table 2: Parameters of the simple-form model

\subsection{Extraction of the parameters of the simple-form model}

The goal of the system is to provide a full system to extract parameters of the chosen model in a simple way. More precisely, the system has to be able to extract parameters from radiological images. For that, a procedure is studied by the surgeons to make the useful radiological images. To validate the method of extraction, a system has been developed to extract parameters from scanner images.

The proposed approach consists in the automatic (or sometimes supervised) extraction of relevant parameters from various medical images (CT slices, X-ray images, radiological images).

\subsubsection{Extraction from CT slices}

In this following example we show the extraction of the humerus retrotorsion angle. The measure of the retrotorsion angle consists in measuring the angle between the humeral head axis and the epicondylar axis, this measure is made automatically, our soft made scrolling CT-scans of the patient with some processing. Afterward, the cards of the distances of Danielsson allow us to determine on all CT-scans a maximum radius which defines the humeral head as well as the minimum radius representing the humeral cylinder. We joint these two centers in order to determine the axis of the humeral head. The last slice of the CT-scans which finished at the elbow allows us to define epicondylar axis. By means of these two lines, we define the retrotorsion angle. 

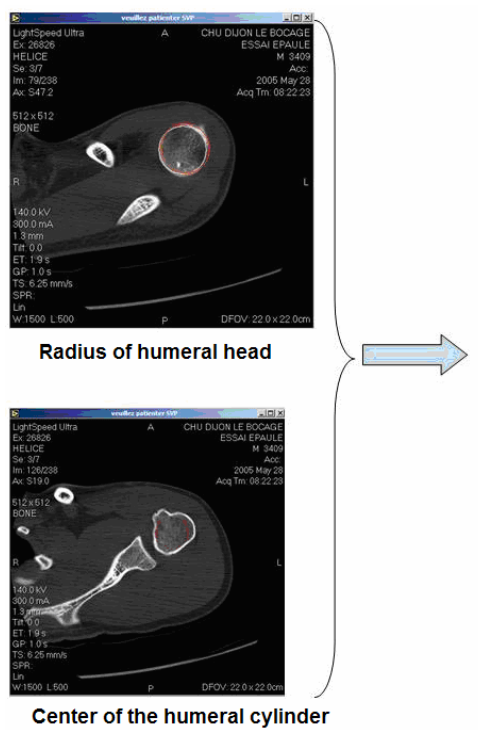

Figure 4. Extraction of retrotorsion angle from CT slice.
Axis of the humeral

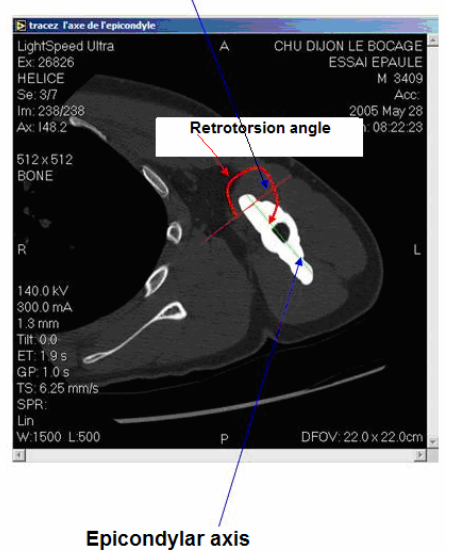

The following table resume the extraction of some parameters (semiautomatic extraction) from CT slice :

Parameters
- Extraction of luminance.
- Thresholding.
- Mathematical morphology
operations (isolate in the
image the interest areas:
particle filter and holes-
filling).
Width of the glenoid cavity:
- The glenoid is extracted
from the image.
$-\quad$ Computes the extremities
along a direction (blue points
in the figure).
$-\quad$ The line joining these
extremities is drawn, and the
center is computed (red point).
- The center of the scapula is
estimated by detecting the
center of mass of the scapula.
- After the others process,
finaly the Width of the glenoid
cavity is computed (half of the
white line along the glenoid
cavity on the last figure).




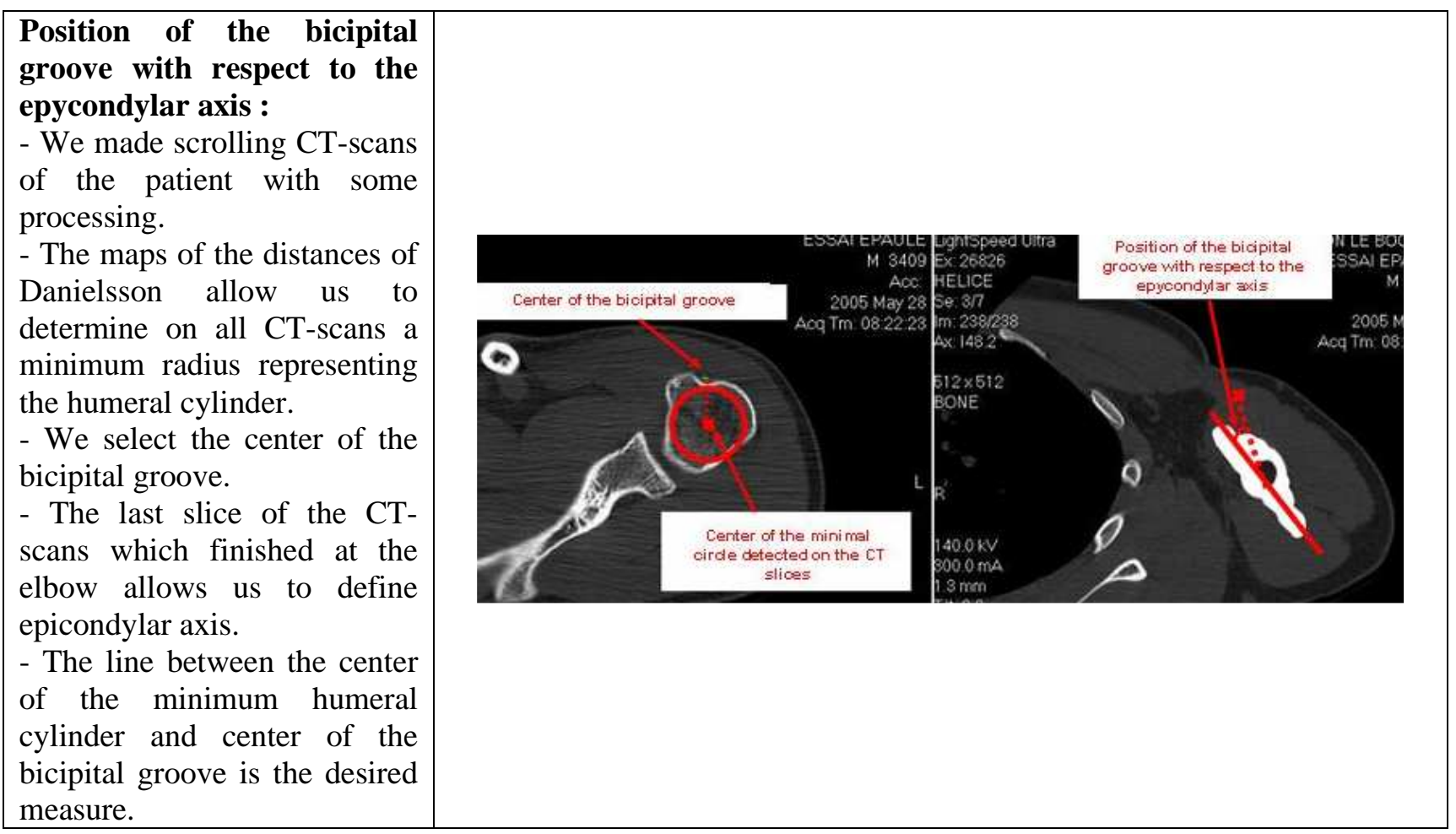

Table 3: Some relevant parameters extracted from CT slices.

All others extraction of parameters are supervised.

\subsubsection{Extraction from the $\mathrm{X}$-ray images}

In this following example we show the extraction of the humeral head. We have radiographic images in JPEG. These Xrays allow extracting the wished parameters. A judicious choice of the plans of radiographic grips is necessary. The extraction is made in a supervised and semiautomatic way, because the parts composing the joint overlap, what makes difficult the automatic extraction of the parameters which interest us. Furthermore, due to the bad quality of the luminosities, operations of treatment of image can be very complex. The measure of radius of the humeral head will be made in supervised way. The user will have to click three points the edge of the humeral head. The measure of the desired radius will be made in an automatic way from these three points.

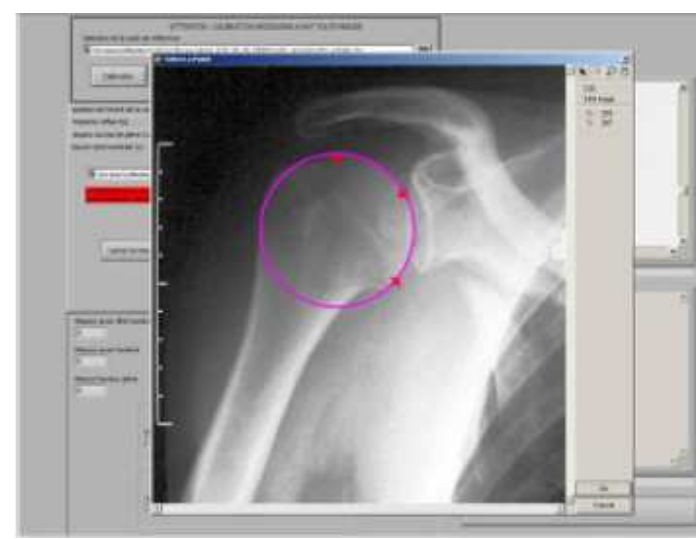

Figure 5. Extraction of the humeral radius from X-ray images. 
The following table resume the extraction of some parameters (supervised and semiautomatic extraction) from X-ray :

\begin{tabular}{l}
\multicolumn{1}{|c|}{ Parameters } \\
\hline Rotation angle of the plane carrying the coracoid \\
with respect to the vertical : \\
- We chose the back view of X-rays shoulder. \\
- We draw three lines. \\
- The software measures the two desired angles.
\end{tabular}

Table 4: Some relevant parameters extracted from X-ray images.

All others extraction of parameters are supervised.

\subsection{Validation of our model}

In order to validate our simple-form model, we have register this model with a humeral and scapula models from 3D scan (clouds of points from resine bone and real bone). The surgeon considered that errors are not strategic for future application of the model. The registration is made by software called RapidForm, after choose 3 points from our two models.

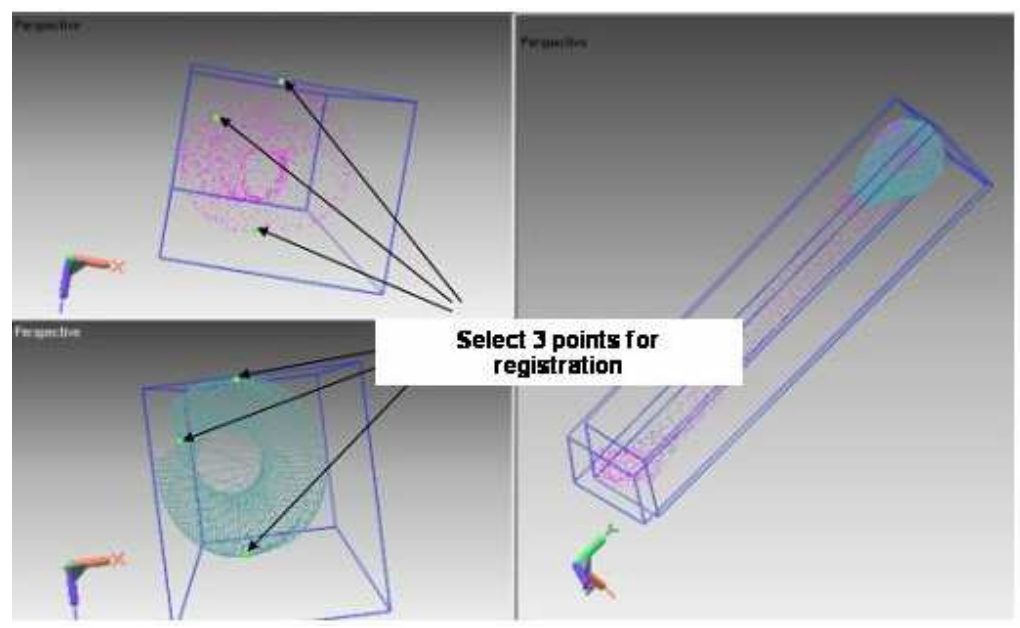

Figure 6. Registration of our humeral model (in green) and numerical model from 3D scan (in pink). 


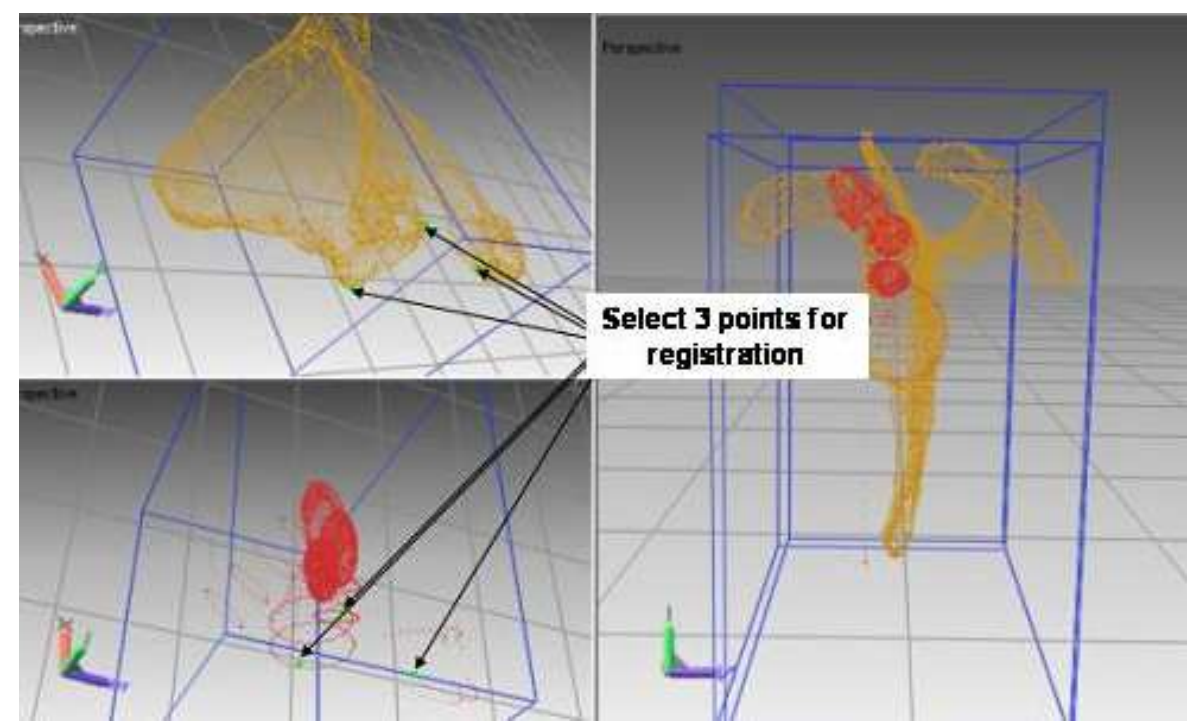

Figure 7. Registration of our scapula model (in red) and numerical model from 3D scan (in brown).

\section{For humerus :}

We mesured the error between a humeral model and our humeral parametric model equivalent. The figure 8 show this error in the histogram error. The error on the humeral head is arround $1 \mathrm{~mm}$ (the blue color), for the surgeon this error are not important. The others parts of the humerus are not important in the operating process.
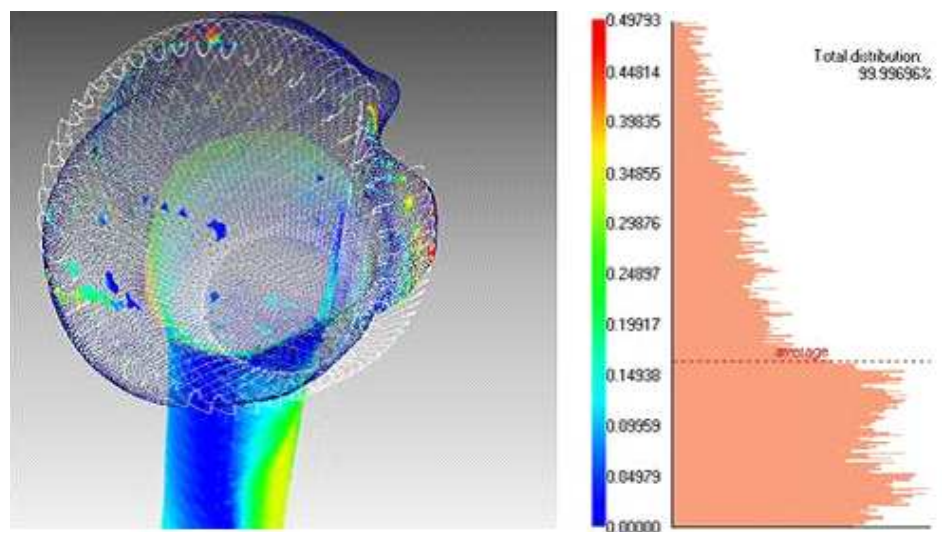

Figure 8. The error between our humeral model and its equivalent numerical model from 3D scan (right), histogram of the error (left).

\section{For scapula :}

We mesured the error between a scapula model and our scapula parametric model equivalent. The figure 9 show this error in the histogram error. The error on the glenoid area is arround $2 \mathrm{~mm}$ (the blue color), for the surgeon this error are not important. The others parts of the scapula are not important in the operating process. 


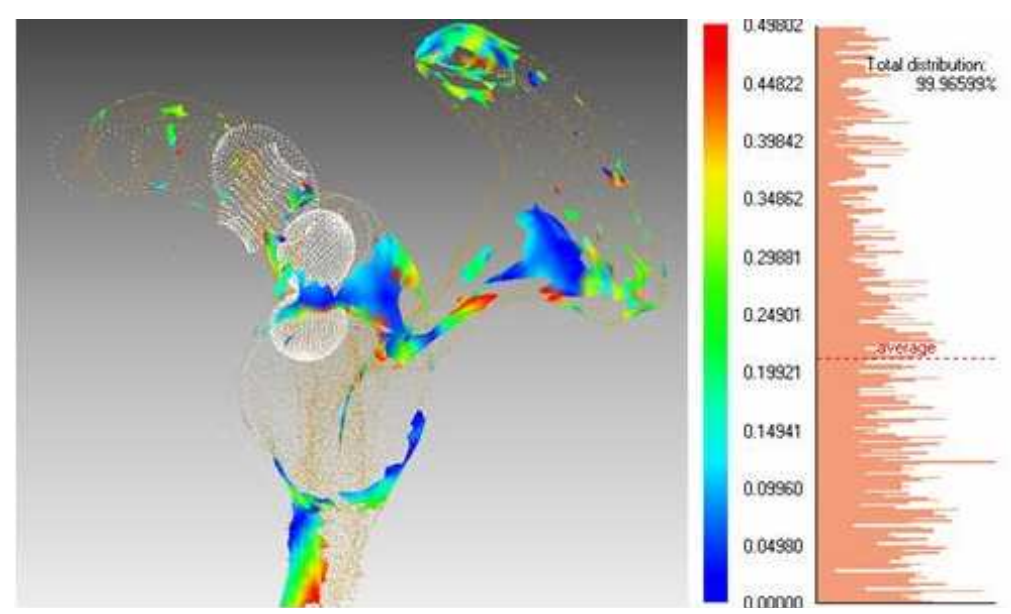

Figure 9. The error between our scapula model and its equivalent numerical model from 3D scan (right), histogram of the error (left).

\section{Pre operative simulation}

The digital mock-up of the shoulder articulation with the CAD model of the prosthesis is used to perform a simulation of the surgery operation.

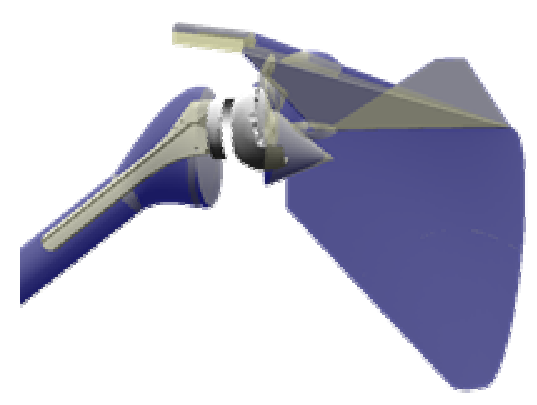

Figure 10. Our simple-form model and CAD of the prosthesis.

A collision detection algorithm between the prosthesis and bones is added in order to measure the mobility of the articulation with respect to the position of the prosthesis and the morphology of the patient. The optimal positioning of the prosthesis can also be deduced from that. Logical operations as subtraction or intersection can be easily computed on this simple model so that the drilling and cutting operations can be simulated on the model and a virtual operation can be considered with real time performance in a PC calculator (Fig. 11). 


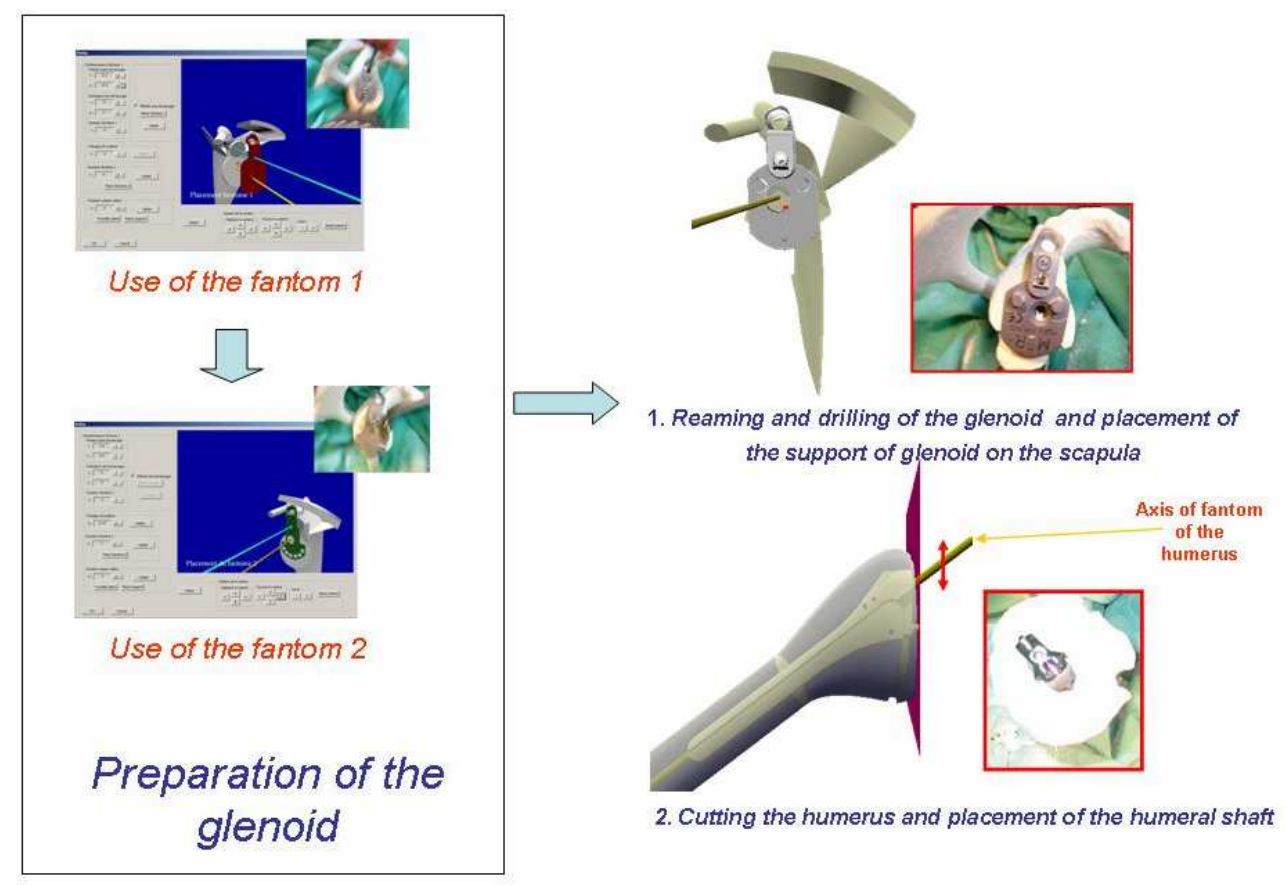

Figure 11. Different steps of the prosthesis placement.

According to the placement of the prosthesis in the bones, the movements of prosthesis articulation can be simulated as shown in the following figures (Fig. 12). The simulation of the movements helps the surgeon to optimise rotation torque of the arm.

In function of the relative position of the prosthesis in the bones, the surgeon can verify the mobility and the feasibility of his operation. Note that the position of the support of the glenoid can also be changed to obtain other mobility results.

Hence, the surgeon can test the best position of the prosthesis, propose different scenarios and save them in order to use them in real-time during the operation.

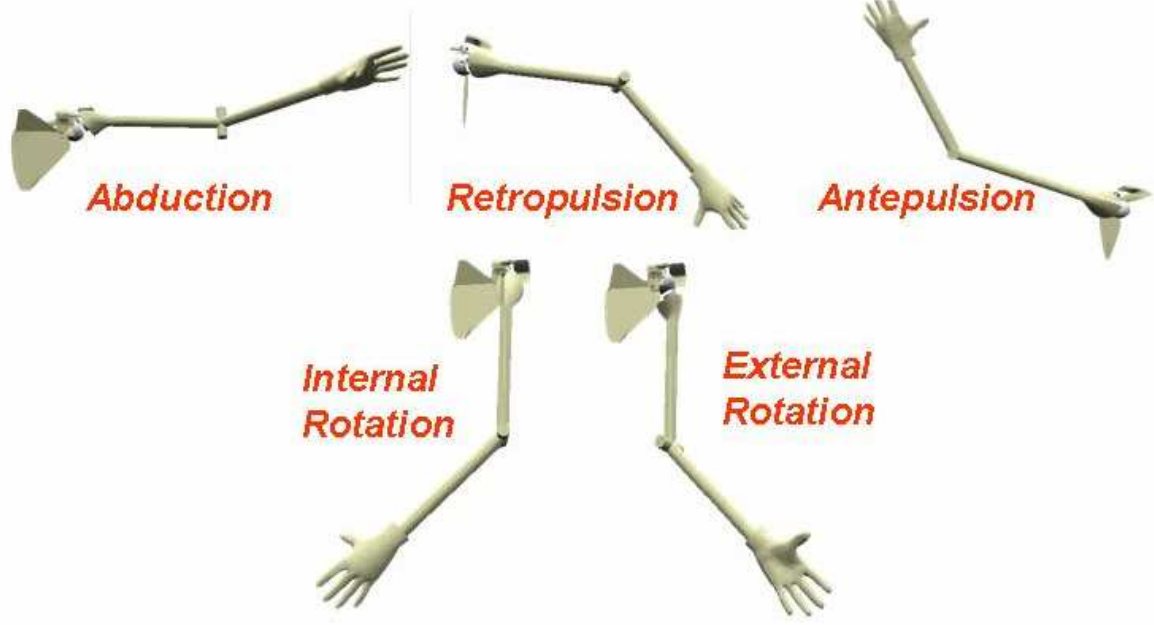

Figure 12. Movements of the prosthetic shoulder.

The Table 5 is an example of results that can be obtained when the cutting plane and drilling axes is moved. This table compares the maximal movements of healthy shoulder, pathological shoulder, prosthetic shoulder with reversed DELTAIII ${ }^{\mathrm{TM}}$ and results obtained with our soft by using inversed DUONCETRIC ${ }^{\mathrm{TM}}$. 


\begin{tabular}{|c|c|c|c|c|}
\hline Kind of movement & $\begin{array}{l}\text { Healthy } \\
\text { shoulder }\end{array}$ & $\begin{array}{c}\text { Case of } \\
\text { pathological } \\
\text { shoulder [26] } \\
\text { (In preoperatory) }\end{array}$ & $\begin{array}{c}\text { Prosthesised } \\
\text { shoulder with } \\
\text { reversed } \\
\text { DELTAIII } \\
{[26]}\end{array}$ & $\begin{array}{c}\text { Prosthesised shoulder with } \\
\text { inversed DUOCENTRIC } \\
\text { Humeral plane at } 1.3 \mathrm{~cm} . \\
\text { Position of drilling to fantom } 1 \text { at } \\
\text { (-0.6cm,-0.1cm). } \\
\text { Depth reaming glenoid with } 0.1 \mathrm{~cm} .\end{array}$ \\
\hline Abduction & $70^{\circ}(\mathrm{GH})$ & $30^{\circ}$ up to $60^{\circ}$ & $80^{\circ}$ up to $180^{\circ}$ & $92^{\circ}(\mathrm{GH})$ \\
\hline Retropulsion & $50^{\circ}$ & ND & ND & $84^{\circ}$ \\
\hline Antepulsion & $160^{\circ}$ & ND & ND & $118^{\circ}$ \\
\hline Internal Rotation & $95^{\circ}$ & $10^{\circ}$ up to $60^{\circ}$ & $40^{\circ}$ up to $80^{\circ}$ & $76^{\circ}$ \\
\hline External Rotation & $80^{\circ}$ & $30^{\circ}$ up to $80^{\circ}$ & $0^{\circ}$ up to $35^{\circ}$ & $82^{\circ}$ \\
\hline
\end{tabular}

Table 5: Results \& comparison with other studies

The trunk and deltoid muscle are not modelled which leads to erroneous results. In our future work, the trunk will be modelled as an obstacle and deltoid as a muscle.

\section{Conclusion}

The paper presents a guided tool for the planning of prosthesis surgical operation. Our goal was to develop a model of shoulder specifically designed for the application of the replacement of shoulder articulation by prosthesis. The model, validated by the surgeon, is based on the use of simple forms. Then, unnecessary details of the articulation for this application, which are strongly dependant of the patient, are not represented. The model is adapted for each patient because of its parameters which can be extracted from scanner images or radiological images. The model is very light and can be manipulated very easily. Virtual surgery can be performed interactively.

As a future work, the 3D model and virtual surgery system will be integrated into a real-time augmented reality device which will be constituted of the spatial positioning of the patient (bones) and surgeon (tools), the registration of the simple model and the real bones of the patient and the development of an ergonomic control interface. If some parameters of our models are fixed by an anatomical study we could reduce the parameters the models. The modeling of the trunk, muscles and tandons wich allow us to use the anatomical DUOCENTRIC ${ }^{\mathrm{TM}}$. This simple form modeling can be used to modeling others articulations or organs 


\section{References}

[1] Alain Cavaille, "Résultat d'une prothèse d'épaule compatible avec une destruction totale de la coiffe des rotateurs", PhD thesis, Dijon university, France, 1993.

[2] AL. Martel, O. Heid, M. Slomczykowski, R. Kerslake, LP. Nolte, "Assessment of 3-dimensional magnetic resonance imaging fast low angle shot images for computer assisted spinal surgery", Computer Aided Surgery, 3:40-44, 1998.

[3] N. Suhm, A. Simeria, R. Hügli, "MR-imaging of bone cements as a first step towards MR-guided vertebroplasty", Proceedings of the Third Annual Meeting of the International Society for Computer Assisted Orthopaedic Surgery (CAOSInternational), Marbella, Spain, June 2003.

[4] AL. Jacob, P. Messmer, A. Kaim, N. Suhm, P. Regazzoni, B. Baumann, "A whole-body registration free navigation system for image-guided surgery and interventional radiology", Invest Radiol, 35:279$288,2000$.

[5] P. Messmer, AL. Jacob, E. Fries, "Technologieintegration und Prozessmanagement - Konzept und Implementierung einer neuartigen Plattform für einzeitige Diagnostik und Therapie des akut Kranken und Verletzten sowie für elektive computerassistierte Chirurgie (CAS)", Unfallchirurg, 104:10251030, 2001.

[6] R. Hofstetter, M. Slomczykowski, Y. Bourquin, LP. Nolte, Fluoroscopy based surgical navigation concept and clinical applications, Computer Assisted Radiology and Surgery, Proceedings of the 11th International Symposium and Exhibition (CAR'97), Germany, 1997.

[7] L. Joskowicz, C. Milgrom, A. Simkin, L. Tockus, Z. Yaniv, "FRACAS: A system for computer-aided image-guided long bone fracture surgery", Comput Aided Surg, 3(6):271-288, 1998.

[8] KT. Foley, DA. Simon, YR. Rampersaud, “ Virtual fluoroscopy: Computer-assisted fluoroscopic navigation”, Spine, 26:347-351, 2001.

[9] M. Heiland, D. Schulze, G. Adam, R. Schmelzle, "3D-imaging of the facial skeleton with an isocentric mobile C-arm system (Siremobil Iso-C3D)”, Dentomaxillofac Radiol, 321:21-25, 2003.

[10] M. Sati, HU. Staübli, Y. Bourquin Y, M. Kunz, LP. Nolte, "Real-time computerized in situ guidance system for ACL graft placement", Computer Aided Surgery, 7:25-40, 2002.

[11] M. Fleute, S. Lavalleé, R. Julliard, "Incorporating a statistically based shape model into a system for computer assisted anterior cruciate ligament surgery", Med Imaging Anal, 3:209-222, 1999.

[12] E. Stindel, JL. Briard, P. Merloz, S. Plaweski, F. Dubrana, C. Lefevre, J. Troccaz, “ Bone morphing: 3D morphological data for total knee arthroplasty”, Computer Aided Surgery, 7:156-168, 2002.

[13] W. Maurel, D. Thalmann, "Human shoulder modeling including scapulo-thoracic constraint and joint sinus cones", Computers\&Graphics, 24:203-218, 2000.

[14] F. Boudjemaï, P.B. Enberg, J.-G. Postaire, "Surface modeling by using Self Organizing Maps of Kohonen", IEEE International Conference on Systems, Man and Cybernetics, Washington DC, 3:2418-2423, 2003.

[15] E. Ferley, M. C. Gascuel, D. Attali, "Skeletal reconstruction of branching shapes", Proceedings of implicit surfaces, 127-142, 1996.

[16] T. Kohonen, "Self-organized formation of topologically correct feature maps", Biological Cybernetics, Springer-Verlag, 1982. 
[17] Sandrine Lanquetin, "Etude des surfaces de subdivision : Intersection, précision et profondeur de subdivision", PhD Thesis, Burgundy university, France, 2004.

[18] W.E. Lorensen, H.E. Cline, "Marching cubes: A high resolution 3D construction algorithm", SIGGRAPH, 21(4):163-169, 1987.

[19] S. Muraki, "Volumetric shape description of range data using blobby model", SIGGRAPH, 227-235, 1991.

[20] S. Pontier, "Reconstruction d'objets déformables à l'aide de fonctions implicites", PhD Thesis, Lyon I university, France, 2000.

[21] R.A. Tafihery, "3D object modeling using radial basis function", Research Report, E.P.F.Lausanne, Switzerland, 2003.

[22] B. Couteau, P. Mansat, R. Darmana, M. Mansat, J. Egan, "Morphological and mechanical analysis of the glenoid by 3D geometric reconstruction using computer tomography", Elsevier Clinical Biomechanics, 15(1), 2000.

[23] E. Bittar, N. Tsingos, M. Gascuel, "Automatic Reconstruction of Unstructured 3D Data: Combining a Medical Axis and Implicit Surfaces”, EUROGRAPHICS, 457-468, 1995.

[24] P.Boileau, G.Walch, "The three-dimensional geometry of the proximal humerus", Bones and joint surgery, 79(B):857-865, 1997.

[25] P. Trouilloud, N. Cheynel, M. Benkhadra, O. Trost, H. Atmani, D. Fofi, F. Merienne, E. Baulot, "Modélisation de l'articulation de l'épaule et navigation", $87^{e}$ Congrès de l'Association des orphologistes de Constanta, Roumania, 2005.

[26] L. De Wilde, M. Mombert, P. Van Petegem, R. Verdonk, "Revision of the shoulder replacement with reversed shoulder prosthesis (DELTA III): Report of five cases", Acta orthopaedica,(4): 348-353, Belgium, 2001. 